\title{
A One-Dimensional Model for Many-Electron Atoms in Extremely Strong Magnetic Fields: Maximum Negative Ionization
}

\author{
Raymond Brummelhuis \\ Department of Mathematics \\ Université de Reims, \\ F-51687 Reims, France \\ raymond.brummelhuis@univ-reims.fr \\ Mary Beth Ruskai * \\ Department of Mathematics \\ University of Massachusetts Lowell \\ Lowell, MA 01854 USA \\ bruskai@cs.uml.edu
}

18 December 1998

\begin{abstract}
We consider a one-dimensional model for many-electron atoms in strong magnetic fields in which the Coulomb potential and interactions are replaced by one-dimensional regularizations associated with the lowest Landau level. For this model we show that the maximum number of electrons $N_{\max }$ satisfies a bound of the form $N_{\max }<2 Z+1+c \sqrt{B}$ where $Z$ denotes the charge of the nucleus, $B$ the field strength and $c$ is a constant. We follow Lieb's strategy in which convexity plays a critical role. For the case $N=2$ with fractional nuclear charge, we also discuss the critical value $Z_{c}$ at which the nuclear charge becomes too weak to bind two electrons.
\end{abstract}

*supported by National Science Foundation Grant DMS-94-08903 


\section{Introduction}

It is well-known that systems in strong magnetic fields behave like systems in one-dimension, i.e., a strong magnetic field confines the particles to Landau orbits orthogonal to the field, leaving only their behavior in the direction of the field subject to significant influence by a static potential. Therefore, a better understanding of one-dimensional systems is essential to understanding the behavior of systems in strong magnetic fields. Although many onedimensional systems, including that of a hydrogen atom with a single electron [2, 8], have been thoroughly studied, relatively little is known about multiparticle atoms confined to one dimension.

In this paper we study the question of bounds on the maximum excess negative charge using one-dimensional models of many-electron atoms. Because our goal is an understanding of the behavior in one-dimension, we do not deal with the question of accuracy of our one-dimensional models as approximations to, or reductions from, real 3-dimensional atoms. However, we sketch such a reduction as motivation for the models considered.

There is some question as to the proper replacement for the Coulomb potential in one dimension [8]. The potential $V(x)=1 /|x|$ is so singular that the one-dimensional Hamiltonian $-\Delta-1 /|x|$ is not even essentially self-adjoint. Fortunately, an electron in a Landau orbit is attracted to a nucleus with charge $Z$, not by a potential of the form $-Z /|x|$, but by a regularized potential which is finite at the nucleus. However, the corresponding regularization of the electron-electron interaction is more complicated unless both electrons have zero angular momentum in the direction of the field. In that case, the regularized interaction has a simple form which is the basis for our model.

There is an extensive literature on atoms in magnetic fields. Interest in atoms in extremely strong fields, which began in the 1970's after the discovery of pulsars, has recently been renewed in the 1990's, culminating in the comprehensive work of Lieb, Solovej and Yngvason (LSY) 16, 17, 18, 29. For a discussion of early work on approximations for atoms in extremely strong magnetic fields we refer the reader to the insightful paper of Rau, Mueller, and Spruch [19] and to the review by Ruderman 20. References to later work are given in the introduction to [17] and a summary of the work of LSY [17, 18] is given in [16, 29]. Rigorous work on atoms in magnetic fields began with the work of Avron, Herbst and Simon (AHS) [1, 2, 3]. LSY [16, 17, 18, 29] not only analyzed extensions of Thomas-Fermi the- 
ory in 5 distinct regions, but showed that these regions suffice to give the correct asymptotics for the exact Hamiltonian. In particular, they showed that the maximum number of electrons $N_{\max }(Z, B)$ which can be bound to a nucleus of charge $Z$ in a constant magnetic field of strength $B$ satisfies $\lim \inf N_{\max }(Z, B) / Z \geq 2$ as $Z \rightarrow \infty$ and $B / Z^{3} \rightarrow \infty$. This should be compared with asymptotic neutrality [15, 7, 25, i.e., $\lim _{Z \rightarrow \infty} N_{\max }(Z, 0) / Z=1$, for atoms without magnetic fields. We hope that the analysis of the simple model in this paper is a modest first step toward a better understanding of the mechanism by which extremely strong magnetic fields bind an "extra" $Z$ electrons, as well as the conjectured converse $N_{\max }(Z, B) \leq 2 Z$.

The full 3-dimensional Pauli Hamiltonian for an N-electron atom with nuclear charge $Z$ in a constant magnetic field of strength $B$, acting on $\mathcal{H}^{N}$, the n-fold tensor product of $L^{2}\left(\mathbf{R}^{3}\right) \otimes \mathbf{C}^{2}$, is

$$
\mathbf{H}(N, Z, B, \alpha)=\sum_{j=1}^{N}\left[\left|\mathbf{P}_{j}+\mathbf{A}\right|^{2}+\sigma_{j} \cdot \mathbf{B}-Z /\left|\mathbf{r}_{j}\right|\right]+\sum_{j<k} \alpha /\left|\mathbf{r}_{j}-\mathbf{r}_{k}\right|
$$

where $\mathbf{A}$ is a vector potential such that $\nabla \times \mathbf{A}=\mathbf{B}$ and $\alpha$ is a coupling constant introduced for convenience in discussing scaling. If the spincoupling term is omitted, it often suffices to consider the corresponding scalar Hamiltonian, which we denote $H(N, Z, B, \alpha)$, as an operator acting only on $\left[L^{2}\left(\mathbf{R}^{3}\right)\right]^{N}$ or the "space" portion of $\mathcal{H}^{N}$. We will choose our coordinate system so that the field $\mathbf{B}=(B, 0,0)$ is in the $\mathrm{x}$-direction and the gauge so that $2 \mathbf{A}=\mathbf{B} \times \mathbf{r}$. The Hamiltonian (1) satisfies the scaling relation $\mathbf{H}(N, Z, B, 1)=B \mathbf{H}\left(N, Z B^{-1 / 2}, 1, B^{-1 / 2}\right)$, i.e., we can scale out the field strength by replacing the nuclear charge $Z$ by $Z B^{-1 / 2}$ and reducing the electron-electron interaction by $B^{-1 / 2}$. Alternatively, we could have included the electron charge unit $e$ explicitly and replaced $e$ by $e B^{-1 / 4}$.

We let $E_{0}(N, Z, B, \alpha)$ denote the infimum of the spectrum of the scalar Hamiltonian $H(N, Z, B, \alpha)$ defined above. It is well-known that the spectrum of the "free" Hamiltonian $\left|\mathbf{P}_{j}+\mathbf{A}\right|^{2}$ is $[B, \infty]$, that the spectrum of $\mid \mathbf{P}_{j}+$ $\left.\mathbf{A}\right|^{2}+\sigma_{j} \cdot \mathbf{B}=\left[\sigma_{j} \cdot\left(\mathbf{P}_{j}+\mathbf{A}\right)\right]^{2}$ is $[0, \infty)$ and that the lowest Landau level has energy $B$ with infinite degeneracy indexed by $m=0,1,2,3, \ldots$ corresponding to angular momentum $-m$ quantized in the field direction. Therefore, the continuous spectrum of $H(N, Z, B, \alpha)$ is $\left[B+E_{0}(N-1, Z, B, \alpha), \infty\right)$, i.e., the continuum begins at $B$ plus the ground state energy for $N-1$ electrons. Thus the question of whether or not $H(N, Z, B, \alpha)$ has a bound state is determined 
by whether or not some test function $\Psi$ satisfies

$$
\langle\Psi, H(N, Z, B, \alpha) \Psi\rangle<\left[B+E_{0}(N-1, Z, B, \alpha)\right]\|\Psi\|^{2} .
$$

For the full Hamiltonian $\mathbf{H}(N, Z, B, \alpha)$, the continuous spectrum is $\left[E_{0}(N-\right.$ $1, Z, B, \alpha), \infty)$ which implies that $\mathbf{H}(N, Z, B, \alpha)$ has a bound state if and only if there is a $\Psi \in \mathcal{H}^{N}$ for which

$$
\langle\boldsymbol{\Psi}, \mathbf{H}(N, Z, B, \alpha) \boldsymbol{\Psi}\rangle<E_{0}(N-1, Z, B, \alpha)\|\boldsymbol{\Psi}\|^{2} .
$$

For the remainder of this paper we will omit explicit consideration of spin [although we will be able to draw some conclusions indirectly. See the remark after equation (13).] Our methods cannot handle the $\sigma \cdot \mathbf{B}$ term explicitly and the inclusion of spin in the wave function does not affect the remaining results in any essential way.

We now consider the the 3-dimensional energy minimization problem restricted to functions whose behavior orthogonal to the field is described entirely by product functions in which all electrons are confined to the lowest Landau level, i.e we restrict to N-electron functions $\Psi$ of the form $\Psi_{m_{1} \ldots m_{N}}=$ $\Phi\left(x_{1} \ldots x_{n}\right) \prod_{k=1}^{N} \gamma_{m_{k}}^{B}\left(r_{k}, \theta_{k}\right)$ where

$$
\gamma_{m}^{B}(r, \theta)=[\pi m !]^{-1 / 2} B^{(m+1) / 2} r^{m} e^{-B r^{2} / 2} e^{-i m \theta}
$$

denotes the Landau level with energy $B$ and angular momentum $-m$. (Note that we are using cylindrical coordinates $(x, r, \theta)$ with $r=\sqrt{y^{2}+z^{2}}$ so that $|\mathbf{r}|=\sqrt{x^{2}+r^{2}}$.) Such expectations satisfy

$$
\begin{aligned}
& \left\langle\Psi_{m_{1} \ldots m_{N}}, H(N, Z, B, 1) \Psi_{m_{1} \ldots m_{N}}\right\rangle \\
& \quad=\left\langle\Psi_{m_{1} \ldots m_{N}}, \widehat{H}(N, Z, B, 1) \Psi_{m_{1} \ldots m_{N}}\right\rangle+N B \\
& \quad=B\left\langle\Psi_{m_{1} \ldots m_{N}}, \widehat{H}\left(N, Z B^{-1 / 2}, 1, B^{-1 / 2}\right) \Psi_{m_{1} \ldots m_{N}}\right\rangle+N B
\end{aligned}
$$

where

$$
\widehat{H}(N, Z, B, \alpha)=\sum_{j=1}^{N}\left[-\left|P_{j}^{x}\right|^{2}-Z /\left|\mathbf{r}_{j}\right|\right]+\sum_{j<k} \alpha /\left|\mathbf{r}_{j}-\mathbf{r}_{k}\right| .
$$

For each fixed choice of $m_{1} \ldots m_{N}$ the minimization problem can be reduced to a one-dimensional problem for the Hamiltonian $\widehat{H}_{x}^{m_{1} \ldots m_{N}}(N, Z, B, \alpha)$ in which the kinetic energy has the usual $-\frac{d^{2}}{d x^{2}}$ form and the potentials $1 /\left|\mathbf{r}_{j}\right|$ 
and $1 /\left|\mathbf{r}_{j}-\mathbf{r}_{k}\right|$ are replaced by regularized potentials denoted by $V_{m_{j}}^{B}\left(x_{j}\right)$ and $W_{m_{j}, m_{k}}^{B}\left(\left|x_{j}-x_{k}\right|\right)$ respectively.

$$
\begin{aligned}
V_{m}^{B}(x) & =\iint\left|\gamma_{m}\right|^{2} /|\mathbf{r}| d y d z \\
& =\frac{B^{m+1}}{m !} \int_{0}^{\infty} \frac{r^{2 m} e^{-B r^{2}}}{\sqrt{x^{2}+r^{2}}} r d r \\
& =[m !]^{-1} \int_{0}^{\infty} \frac{u^{m} e^{-u}}{\sqrt{x^{2}+u / B}} d u \\
& =\frac{2 B^{m+1}}{m !} e^{B x^{2}} \int_{|x|}^{\infty}\left(t^{2}-x^{2}\right)^{m} e^{-B t^{2}} d t
\end{aligned}
$$

which satisfies the scaling relation $V_{m}^{B}(x)=\sqrt{B} V_{m}^{1}(\sqrt{B} x)$ as one would expect from the scaling properties of $H(N, Z, B, \alpha) . W_{m_{j}, m_{k}}^{B}$ is defined analogously; we postpone discussion of its explicit form. That the regularizations $V_{m, B}(x)$ are important for atoms in magnetic fields goes back at least to Schiff and Snyder [24] in (1939) and played an important role in the AHS study [2] of hydrogen. In the case $m=0$ and $B=1$, (6) can be rewritten as

$$
\begin{aligned}
V_{0}(x) & =\sqrt{\pi} e^{x^{2}}[1-\operatorname{erf}(x)] \\
& =\int_{0}^{\infty} \frac{e^{-u}}{\sqrt{x^{2}+u}} d u=2 e^{x^{2}} \int_{x}^{\infty} e^{-t^{2}} d t
\end{aligned}
$$

A comparison with the usual Coulomb potential is given in Figure 1.

We now describe the correspondence between a restricted three-dimensional minimization problem for a Hamiltonian with a magnetic field, namely,

$$
E_{0}^{m_{1} \ldots m_{N}}(N, Z, B, \alpha)=\inf _{\Psi_{m_{1} \ldots m_{N}}}\left\langle\Psi_{m_{1} \ldots m_{N}}, H(N, Z, B, \alpha) \Psi_{m_{1} \ldots m_{N}}\right\rangle
$$

and the one-dimensional minimization problem

$$
E_{0, x}^{m_{1} \ldots m_{N}}(N, Z, B, \alpha)=\inf _{\Phi}\left\langle\Phi, \widehat{H}_{x}^{m_{1} \ldots m_{N}}(N, Z, B, \alpha) \Phi\right\rangle
$$

where

$$
\widehat{H}_{x}^{m_{1} \ldots m_{N}}(N, Z, B, \alpha)=\sum_{j=1}^{N}\left[-\frac{d^{2}}{d x_{j}^{2}}-Z V_{m_{j}}^{B}\left(x_{j}\right)\right]+\alpha \sum_{j<k} W_{m_{j}, m_{k}}^{B}\left(\left|x_{j}-x_{k}\right|\right)
$$




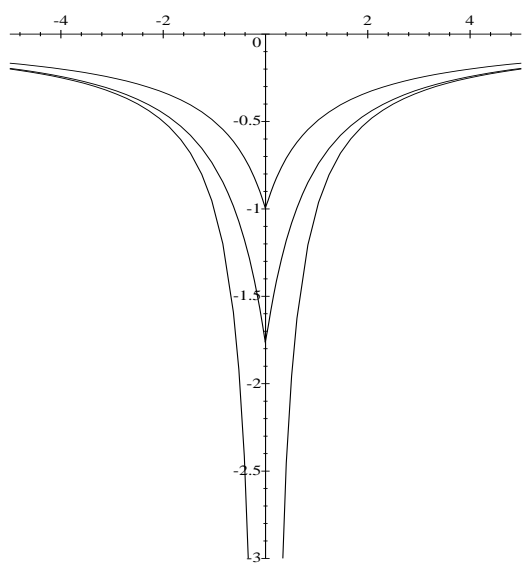

Figure 1: Comparison of the potentials (reading from the top down) $-\frac{1}{|x|+1}$, $-V_{0}(x)$, and $-\frac{1}{|x|}$

in which no magnetic field is explicitly present. Since

$$
\left\langle\Psi_{m_{1} \ldots m_{N}}, H(N, Z, B, \alpha) \Psi_{m_{1} \ldots m_{N}}\right\rangle=\left\langle\Phi, \widehat{H}_{x}^{m_{1} \ldots m_{N}}(N, Z, B, \alpha) \Phi\right\rangle+N B
$$

we can conclude that

$$
\begin{array}{r}
\left\langle\Psi_{m_{1} \ldots m_{N}}, H(N, Z, B, \alpha) \Psi_{m_{1} \ldots m_{N}}\right\rangle>E_{0}^{m_{1} \ldots m_{N-1}}(N-1, Z, B, \alpha)+B \\
\Longleftrightarrow\left\langle\Phi, \widehat{H}_{x}^{m_{1} \ldots m_{N}}(N, Z, B, \alpha) \Phi\right\rangle>E_{0, x}^{m_{1} \ldots m_{N-1}}(N-1, Z, B, \alpha) .
\end{array}
$$

Thus, we have reduced the problem of determining whether or not the somewhat artificial problem of whether or not an atom in magnetic field $B$ whose electrons have prescribed angular momentum corresponding to $m_{1} \ldots m_{N}$ has a bound state to that of whether or not a related one-dimensional system has a bound state. Although, we do not consider spin-coupling explicitly, it does follow from (3) that we can also conclude that

$$
\begin{array}{r}
\left\langle\boldsymbol{\Psi}_{m_{1} \ldots m_{N}}, \mathbf{H}(N, Z, B, \alpha) \boldsymbol{\Psi}_{m_{1} \ldots m_{N}}\right\rangle>E_{0}^{m_{1} \ldots m_{N-1}}(N-1, Z, B, \alpha) \\
\Longleftrightarrow\left\langle\Phi, \widehat{H}_{x}^{m_{1} \ldots m_{N}}(N, Z, B, \alpha) \Phi\right\rangle>E_{0, x}^{m_{1} \ldots m_{N-1}}(N-1, Z, B, \alpha) .
\end{array}
$$

where $\boldsymbol{\Psi}_{m_{1} \ldots m_{N}}$ is chosen so that all components in $\mathbf{C}^{2}$ correspond to spin "down". (This observation, which is a fortuitous consequence of the physical 
fact that the coefficient of $\sigma \cdot \mathbf{B}$ is exactly 1 , was pointed out to us by J.P. Solovej.)

We now consider the regularization of the interaction in the special case in which $m_{j}=m_{k}=0$. Recall that $\gamma_{0}^{B}(y, z)=B \pi^{-1} e^{-B\left(y^{2}+z^{2}\right) / 2}=B \pi^{-1} e^{-B r^{2} / 2}$.

$$
\begin{aligned}
W_{0,0}^{B}\left(\left|x_{1}-x_{2}\right|\right) & =\iint d y_{1} d z_{1} \iint d y_{2} d z_{2} \frac{\left|\gamma_{0}^{B}\left(y_{1}, z_{1}\right)\right|^{2}\left|\gamma_{0}^{B}\left(y_{2}, z_{2}\right)\right|^{2}}{\sqrt{\left(x_{1}-x_{2}\right)^{2}+\mid\left(y_{1}, z_{1}\right)-\left(y_{2}, z_{2}\right)^{2}}} \\
& =B \int_{o}^{\infty} d t \frac{2 t e^{-B t^{2}}}{\sqrt{\left(x_{1}-x_{2}\right)^{2}+2 t^{2}}} \\
& =\frac{1}{\sqrt{2}} V_{0}^{B}\left(\frac{\left|x_{1}-x_{2}\right|}{\sqrt{2}}\right)
\end{aligned}
$$

where we have made the change of variables

$$
\mathbf{s}=\frac{1}{\sqrt{2}}\left[\left(y_{1}, z_{1}\right)+\left(y_{2}, z_{2}\right)\right], \quad \mathbf{t}=\frac{1}{\sqrt{2}}\left[\left(y_{1}, z_{1}\right)-\left(y_{2}, z_{2}\right)\right]
$$

with $s=|\mathbf{s}|, t=|\mathbf{t}|$, and used the fact that

$$
\begin{aligned}
\left|\gamma_{0}^{B}\left(y_{1}, z_{1}\right)\right|^{2}\left|\gamma_{0}^{B}\left(y_{2}, z_{2}\right)\right|^{2} & =B^{2} \pi^{-2} e^{-B\left(r_{1}^{2}+r_{2}^{2}\right)} \\
& =B^{2} \pi^{-2} e^{-B\left(s^{2}+t^{2}\right)}=\left|\gamma_{0}^{B}(\mathbf{s})\right|^{2}\left|\gamma_{0}^{B}(\mathbf{t})\right|^{2} .
\end{aligned}
$$

Thus, the exceedingly simple relation $W_{0,0}^{B}\left(\left|x_{1}-x_{2}\right|\right)=2^{-1 / 2} V_{0}^{B}\left(2^{-1 / 2}\left|x_{1}-x_{2}\right|\right)$ follows from the invariance of $\left|\gamma_{0}^{B}(\mathbf{s})\right|^{2}\left|\gamma_{0}^{B}(\mathbf{t})\right|^{2}$ under the transformation of $\mathbf{s}, \mathbf{t}$ to $(\mathbf{s} \pm \mathbf{t}) / \sqrt{2}$. This unusual invariance, corresponding to the mixing of coordinates of two particles, will not hold if $m \neq 0$. Symmetrizing the product or replacing $\Psi_{m_{1} \ldots m_{N}}$ by an arbitrary element of the projection onto the lowest Landau level, would require consideration of exchange terms as well. Therefore, we will only study models corresponding to constraining all electrons to have angular momentum zero. Since $\Psi_{0 \ldots 0}$ is then symmetric with respect to exchange of $\left(y_{j}, z_{j}\right)$ with $\left(y_{k}, z_{k}\right)$, it will have the same permutational symmetry as $\Phi\left(x_{1} \ldots x_{N}\right)$.

Despite the severity of the restriction to $m=0$, our model seems wellsuited to study of the bounds on the negative ionization. Integration by parts of (6) easily yields

$$
V_{m+1}^{B}(x) \leq V_{m}^{B}(x) \leq V_{0}^{B}(x) \leq 1 /|x|
$$

with the difference greatest at the origin and all potentials satisfying $V_{m}^{B}(x) \approx$ $1 /|x|$ for large $x$. Moreover, for any choice of $m_{j}, m_{k}$ whenever $\left|x_{j}-x_{j}\right|$ is 
large, $W_{m_{j}, m_{k}}^{B}\left(\left|x_{j}-x_{j}\right|\right) \approx 1 /\left|x_{j}-x_{j}\right|$ as well. Thus it appears unlikely that placing some electrons in Landau levels with $m \neq 0$ will allow binding if $m=0$ does not.

With this heuristic background, we study models for N-electron atoms in one-dimension corresponding to Hamiltonians of the form

$$
\begin{aligned}
h(N, Z, M) & =M \widehat{H}_{x}^{0 \ldots 0}\left(N, Z, M^{-2}, 1\right) \\
& =\sum_{j=1}^{N}\left[-\frac{1}{M} \frac{d^{2}}{d x^{2}}-Z V_{0}\left(x_{j}\right)\right]+\sum_{j<k} \frac{1}{\sqrt{2}} V_{0}\left(\frac{\left|x_{j}-x_{k}\right|}{\sqrt{2}}\right)
\end{aligned}
$$

with the "mass" $M$ proportional to $B^{-1 / 2}$. Because of the scaling relation $H(N, Z, B, 1)=B H\left(N, Z B^{-1 / 2}, 1, B^{-1 / 2}\right)$, the only role of the field strength in the one-dimensional situation, is to reduce the mass by a factor of $B^{-1 / 2}$. Thus, for simplicity, we have set both $B=1$ and the coupling constant $\alpha=1$ leaving the field strength implicit in the mass $M$.

As observed in [2], the regularized potentials satisfy

$$
\frac{1}{\sqrt{(m+1) B}+|x|} \leq \frac{1}{\sqrt{(m+1) B+|x|^{2}}} \leq V_{m}^{B}(x) \leq \frac{1}{|x|} .
$$

The cut-off potential $V_{\text {cut }}(x)=1 /(|x|+1)$ is also of some interest. Haines and Roberts [8] have given explicit solutions to the eigenvalue problem for the hydrogenic Hamiltonian $-\Delta-V_{\text {cut }}(x)$. By the above remark, $V_{\text {cut }}(x) \leq$ $V_{0}^{1}(x) \leq 1 /|x|$.

In sections 2 and 3, we study Hamiltonians of the form (16) in the two cases $V=V_{\text {cut }}$ and $V=V_{0}$. For both models we show that the existence of a bound state implies $N<2 Z+1+c \sqrt{B}$.

\section{Two-electron systems}

We now discuss in more detail the behavior of the one-dimensional Hamiltonian (16) when the number of electrons is $N=2$. Although our discussion is descriptive and non-rigorous, we believe the insights are useful. The twoelectron Hamiltonian can be written in the form.

$$
h\left(2, Z, B^{-1 / 2}\right)=-\sqrt{B}\left[\frac{d^{2}}{d x_{1}^{2}}+\frac{d^{2}}{d x_{2}^{2}}\right]+W\left(x_{1}, x_{2}\right)
$$


where

$$
W\left(x_{1}, x_{2}\right)=-Z V_{0}\left(x_{1}\right)-Z V_{0}\left(x_{2}\right)+\frac{1}{\sqrt{2}} V_{0}\left(\frac{\left|x_{1}-x_{2}\right|}{\sqrt{2}}\right)
$$

Consider a classical system of three particles on a line with charges -1 , $+Z,-1$ interacting with the usual inverse square Coulomb force (or, equivalently, $q_{i} q_{j} /\left|x_{i}-x_{j}\right|$ potential). The only potentially stable configuration is one in which the particles are arranged symmetrically with the two negatively charged particles on opposite sides of, and the same distance from, the positive "nucleus" as shown in Fig. 1. Even in this case, the system is stable only for $Z=1 / 4$. For $Z<1 / 4$, both "electrons" will move off to infinity, while for $Z>1 / 4$, the "electrons" collapse into the center. This suggests that for $Z>1 / 4$ one might be able to show that the system binds by using a trial function $\Psi_{a}$ in which the two electrons are localized on opposite sides of the nucleus at a distance $a$ sufficiently far from the center that $V_{0}( \pm a) \approx 1 / a$. (Permutational symmetry is not relevant; if such a trial function binds, then a bosonic system will also bind.) This picture would require that $\Delta x_{i}<a$ for each electron so that, by the uncertainty principle, the kinetic energy satisfies $\left\langle\Psi_{a}\left[-\frac{d^{2}}{d x_{1}^{2}}\right] \Psi_{a}\right\rangle>1 / a^{2}$. Thus we estimate

$$
\left\langle\Psi_{a} h\left(2, Z, B^{-1 / 2}\right) \Psi_{a}\right\rangle>2 \frac{\sqrt{B}}{a^{2}}-\frac{2 Z}{a}+\frac{1}{2 a} .
$$

Minimizing over $a$ yields $a_{\min }=2 \sqrt{B} /\left(Z-\frac{1}{4}\right)$ and

$$
\left\langle\Psi_{a} h\left(2, Z, B^{-1 / 2}\right) \Psi_{a}\right\rangle>-\frac{\left(Z-\frac{1}{4}\right)^{2}}{2 \sqrt{B}} .
$$

The continuous spectrum begins at the ground state energy of the corresponding one-electron Hamiltonian $h\left(1, Z, B^{-1 / 2}\right)=-\sqrt{B} \frac{d^{2}}{d x^{2}}-Z V_{0}(x)$ which Avron, Herbst and Simon [2] showed is given asymptotically (for large $B$ ) by $E_{0}\left(1, Z, B^{-1 / 2}\right)=-\frac{Z^{2}}{\sqrt{B}}\left(\log \frac{Z^{2}}{\sqrt{B}}\right)^{2}$ Thus, binding would require

$$
\left(Z-\frac{1}{4}\right)^{2}>2 Z^{2}\left(\log \frac{Z}{\sqrt{B}}\right)^{2} .
$$

This is obviously false for large $B$, which suggests that the uncertainty principle prevents the corresponding one-dimensional system from binding, even 

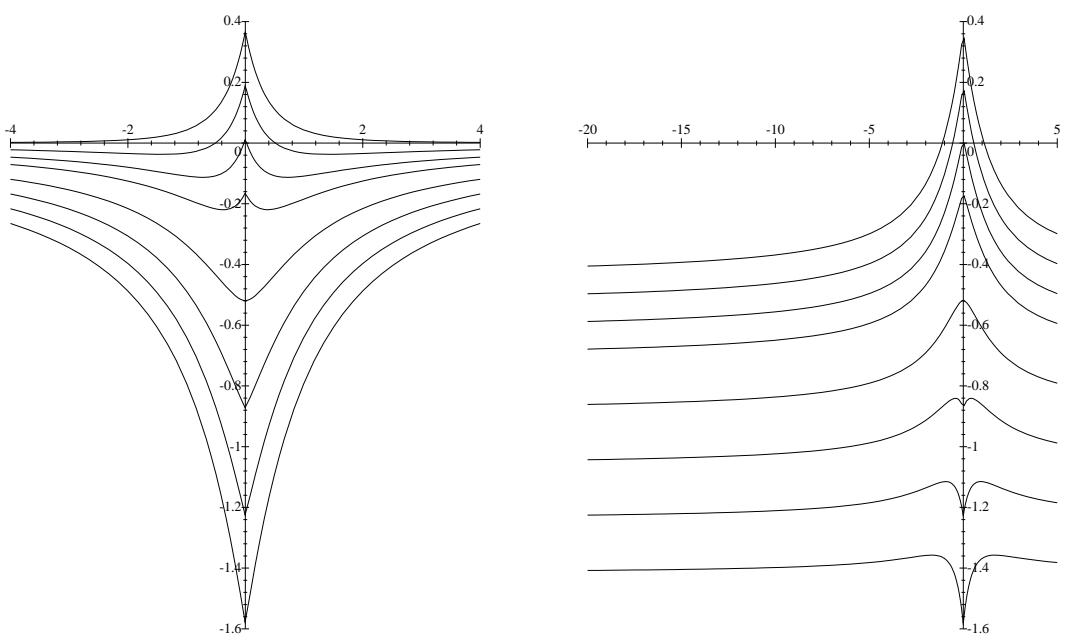

Figure 2: $W(x,-x)$ and $W(x, 0)$ for (reading from the top down) $Z=0.25,0.3,0.35,0.4,0.5,0.6,0.7,0.8$. The left graph, $W(x,-x)$, describes the potential when the electrons are on opposite sides of the nucleus with each a distance $x$ away. The right graph, $W(x, 0)$, describes the potential when one electron is fixed at the origin; the left asymptote then corresponds to the other electron moving to infinity.

when the classical system collapses. Because $M \sim B^{-1 / 2}$, large field strength corresponds to small mass, i.e., as the field strength increases, the electrons become "lighter" and more difficult to localize. Thus we must seek a different mechanism to explain binding of a one-dimensional two-electron atom.

Fortunately, our model provides an alternative mechanism for binding. The regularization of the potential at the origin combined with the effective reduction of the interaction by $1 / \sqrt{2}$, permits both electrons to be close to the nucleus for $Z>1 / 2^{3 / 2}$ in the sense that $-2 Z V_{0}(0)+2^{-1 / 2} V_{0}<0$. Of course, the uncertainty principle also precludes binding with a trial function in which both electrons are exactly at the center. Nevertheless, we believe that the mechanism for binding is that the effective reduction in the interaction permits the two electrons to overlap strongly near the nucleus.

The Hamiltonian (18) can be regarded as describing either two particles in one-dimension or one particle in the field of the two-dimensional potential $W(x, y)$ given by (19). Regardless of our viewpoint, the continuous spectrum will begin at the AHS [2] estimate of $-\frac{Z^{2}}{\sqrt{B}}\left(\log \frac{Z^{2}}{\sqrt{B}}\right)^{2}$. Since this is 
close to zero for large $B$, we will regard $W(x, y)$ as attractive where it is negative and repulsive where it is positive. One might expect $W(x, y)$ to have its minimum on the line $y=-x$, corresponding to electrons on opposite sides of the nucleus. The actual situation, which is described below, is more complex. Plots of $W(x,-x)$ and $W(x, 0)$ are shown in Fig. 2 for $Z=0.25,0.3,0.35 \approx \frac{1}{2 \sqrt{2}}, 0.4,0.5,0.6,0.7 \approx \frac{1}{\sqrt{2}}, 0.8$. The graph of $W(x, 0)$ is deliberately asymmetric so that the left asymptote, $W(-\infty, 0)$ can be compared to the minimum of $W(x,-x)$ at $x=0$. We now discuss this behavior in more detail, noting that all the qualitative features can also be verified analytically.

- $\frac{1}{4}<Z<\frac{1}{2 \sqrt{2}}$ : The potential does have a pair of weak minima along the line $y=-x$; however, closer examination of the full two-dimensional potential shows that these are not true minima, but saddle points for $W(x, y)$. The potential is repulsive at the origin and only weakly attractive elsewhere.

- $\frac{1}{2 \sqrt{2}}<Z<\frac{1}{2}$ : The potential is now attractive at the origin. However, as above, the two weak minima on the line $y=-x$ correspond to saddle points of $W(x, y)$.

- $\frac{1}{2}<Z<\frac{1}{\sqrt{2}}$ : The two weak minima on the line $y=-x$ have now coalesced into a true (two-dimensional) minimum at the origin. However, this is only a local minimum. $W(0,0)>W(0, \infty)$ so that the energy will decrease if one of the particles remains at the origin while the other goes off to infinity. Fig. 2 suggests that the minimum along the $x=0$ and $y=0$ lines is too shallow to prevent one of the electrons from tunnelling through to infinity. Thus, we expect resonances, but not bound states in this region.

- $Z>\frac{1}{\sqrt{2}}$ : The potential has a true minimum at the origin.

Thus, the behavior of the potential $W(x, y)$ strongly suggests that, at least for sufficiently large $B$, binding occurs for $Z>\frac{1}{\sqrt{2}} \approx 0.7$ and that the mechanism which permits this is the reduction in strength of the repulsion by $1 / \sqrt{2}$ which permits both electrons to simultaneously sit near the nucleus. The behavior of the regularized potential model for a two-electron atom in one dimension seems to be very different from that of classical Coulomb particles confined to a line. The binding cut-off of $Z_{c} \approx 0.7$ represents a very 


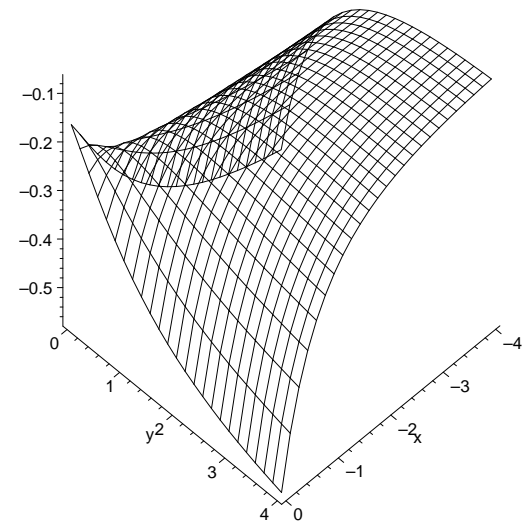

Figure 3: Saddle point of $W(x, y)$ for $Z=0.4$ in the quadrant $x<0, y>0$.

modest "binding enhancement" when compared to the value of $Z_{c} \approx 0.9$ obtained in [4] for a two-electron atom in three dimension in the absence of a magnetic field. [An "enhancement" to the level of $2=N>2 Z+1$ would require $Z_{c}<0.5$.]

\section{$3 \quad$ Kinetic Energy and Lieb's Strategy}

We now describe the elegant strategy used by Lieb [13] (see also section 3.8 of [6]) to show that for real atoms the maximum number of electrons that can be bound is less than $2 Z+1$ irrespective of permutational symmetry. The essence of Lieb's argument is to show that one can replace the variation over N-electron wave functions $\Psi\left(\mathbf{r}_{1}, \ldots \mathbf{r}_{N}\right)$ by a variation over density matrices of the form

$$
\begin{aligned}
& \Gamma\left(\mathbf{r}_{1}, \ldots \mathbf{r}_{N} ; \mathbf{s}_{1}, \ldots \mathbf{s}_{N}\right) \\
& \quad=\sum_{j=1}^{N} \overline{\left[\nu\left(\mathbf{r}_{j}\right)\right]^{1 / 2} \Psi\left(\mathbf{r}_{1}, \ldots \mathbf{r}_{N}\right)}\left[\nu\left(\mathbf{s}_{j}\right)\right]^{1 / 2} \Psi\left(\mathbf{s}_{1}, \ldots \mathbf{s}_{N}\right)
\end{aligned}
$$

where $\nu(\mathbf{r})>0$ is a strictly positive function which will be chosen later. Note that, even though the functions $\Psi \nu_{j}^{1 / 2}$ are not orthonormal, $\operatorname{Tr} \Gamma A=$ $\sum_{j}\left\langle\left[\nu\left(\mathbf{r}_{j}\right)\right]^{1 / 2} \Psi, A\left[\nu\left(\mathbf{r}_{j}\right)\right]^{1 / 2} \Psi\right\rangle$ for any operator $A$. Despite the introduction 
of the function $\nu$, which will be chosen later, the $\Psi$ used in $\Gamma$ satisfies the same symmetry or domain constraints as the original minimization problem.

It will be useful to describe Lieb's argument in the rather general situation of an N-particle Hamiltonian, $H_{N}$ with the structure $H_{N}=H_{N-1}^{j}+K_{j}+V_{\text {int }}^{j}$ where $K_{j}=\left|\mathbf{P}_{j}+\mathbf{A}\right|^{2}$ denotes the kinetic energy, $H_{N-1}^{j}$ is the $N$-1-electron Hamiltonian in which the j-th electron is omitted and $V_{\text {int }}^{j}$ is a potential which describes the interaction of the $\mathrm{j}$-th electron with the rest of the system. Lieb studied $H_{N}=H(N, Z, 0,1)$ while we will consider $H_{N}=h(N, Z, M)$. In our case,

$$
V_{\mathrm{int}}^{j}=-Z V\left(x_{j}\right)+\sum_{k \neq j} 2^{-1 / 2} V\left(2^{-1 / 2}\left|x_{j}-x_{k}\right|\right)
$$

Now assume that $H_{N}$ actually has a bound state so that there is a $\Psi_{0}$ satisfying $H_{N} \Psi_{0}=E_{0} \Psi_{0}$. This implies that for any function $\nu$ and any choice of $j$,

$$
\nu\left(\mathbf{r}_{j}\right) H_{N} \Psi_{0}\left(\mathbf{r}_{1}, \ldots \mathbf{r}_{N}\right)=E_{0} \nu\left(\mathbf{r}_{j}\right) \Psi_{0}\left(\mathbf{r}_{1}, \ldots \mathbf{r}_{N}\right)
$$

as well. Hence

$$
\left\langle\Psi_{0}, \nu\left(\mathbf{r}_{j}\right) H_{N} \Psi_{0}\right\rangle=E_{0}\left\langle\Psi_{0}, \nu\left(\mathbf{r}_{j}\right) \Psi_{0}\right\rangle .
$$

Then if $\Gamma_{0}$ is the density matrix of the above form corresponding to the ground state $\Psi_{0}$, and $\nu_{j}$ denotes $\nu\left(\mathbf{r}_{j}\right)$

$$
\begin{aligned}
& E_{0}(N) \operatorname{Tr} \Gamma_{0}=E_{0}(N) \sum_{j=1}^{N}\left\langle\Psi \nu_{j} \Psi\right\rangle \\
& \quad=\operatorname{Tr} \Gamma_{0} H_{N}+\sum_{j=1}^{N}\left[\left\langle\Psi \nu_{j} K_{j} \Psi\right\rangle-\left\langle\nu_{j}^{1 / 2} \Psi K_{j} \nu_{j}^{1 / 2} \Psi\right\rangle\right] \\
& \quad=\sum_{j}\left\langle\nu_{j}^{1 / 2} \Psi,\left[H_{N-1}^{j}+V_{\text {int }}^{j}\right] \nu_{j}^{1 / 2} \Psi\right\rangle+\sum_{j=1}^{N}\left\langle\Psi \nu_{j} K_{j} \Psi\right\rangle \\
& \geq E_{0}(N-1) \operatorname{Tr} \Gamma_{0}+\sum_{j}\left[\left\langle\nu_{j}^{1 / 2} \Psi, V_{\text {int }}^{j} \nu_{j}^{1 / 2} \Psi\right\rangle+\left\langle\Psi \nu_{j} K_{j} \Psi\right\rangle\right]
\end{aligned}
$$

The inequality in (27) above follows from the variational principle for $H_{N-1}^{j}$. Although $\left\langle\Psi \nu_{j} K_{j} \Psi\right\rangle$ need not be real in general, a careful analysis of the 
argument above shows that when $\Psi$ is an eigenstate of $H_{N}$ it is real because it can be written as the difference of two real quantities. In Lieb's original formulation, $\nu$ was chosen so that this real quantity satisfied $\left\langle\Psi \nu_{j} K_{j} \Psi\right\rangle>0$. Then if $\sum_{j}\left\langle\nu_{j}^{1 / 2} \Psi V_{\text {int }}^{j} \nu_{j}^{1 / 2} \Psi\right\rangle>0$ it follows that $E_{0}(N) \operatorname{Tr} \Gamma_{0}>E_{0}(N-1) \operatorname{Tr} \Gamma_{0}$ which contradicts the requirement for binding of $E_{0}(N)<E_{0}(N-1)$. This reduces the problem of showing that $H(N, Z, 0, \alpha)$ has no bound states in the absence of magnetic fields to that of showing that $\sum_{j}\left\langle\nu_{j}^{1 / 2} \Psi V_{\text {int }}^{j} \nu_{j}^{1 / 2} \Psi\right\rangle>0$. However, in a constant magnetic field the continuum begins at $B+E_{0}(N-1)$ so that a similar reduction would require the stronger condition $\left.\left\langle\Psi \nu_{j} K_{j} \Psi\right\rangle\right\rangle$ $B$.

In order to study $\left\langle\Psi \nu_{j} K_{j} \Psi\right\rangle$ in more detail in different situations, it is convenient to write $\nu_{j}$ as $g^{2}$ and observe that formally

$$
\begin{gathered}
\left\langle\Psi g^{2},\left|i \nabla_{j}+\mathbf{A}\right|^{2} \Psi\right\rangle=\left\langle\left[i \nabla_{j}+\mathbf{A}\right](g \Psi) g,\left[i \nabla_{j}+\mathbf{A}\right](g \Psi) g^{-1}\right\rangle \\
=\left\langle\left[i \nabla_{j}+\mathbf{A}\right] g \Psi,\left[i \nabla_{j}+\mathbf{A}\right](g \Psi)\right\rangle-\int|g \Psi|^{2}\left|\frac{\nabla g}{g}\right|^{2} \\
\quad \pm 2 i \Re\left\langle\Psi \nabla g,\left[i \nabla_{j}+\mathbf{A}\right](g \Psi) .\right\rangle
\end{gathered}
$$

Note that the localization error, $-\int|g \Psi|^{2}\left|\frac{\nabla g}{g}\right|^{2}=\int|g \Psi|^{2} \nabla g \cdot \nabla\left(g^{-1}\right)$, shows a certain symmetry between $g$ and $g^{-1}$. If we now let $g=\nu^{1 / 2}$, (28) implies

$$
\Re\left\langle\Psi \nu_{j} K_{j} \Psi\right\rangle=\left\langle\Psi \nu_{j}^{1 / 2},\left[K_{j}-\left|\frac{\nabla_{j} \nu_{j}}{2 \nu_{j}}\right|^{2}\right] \Psi \nu_{j}^{1 / 2}\right\rangle .
$$

For the typical choice $\nu=[V(\mathbf{r})]^{-1}$, one has $\nabla \nu(\mathbf{r}) / \nu(\mathbf{r})=-\nabla V(\mathbf{r}) / V(\mathbf{r})$ which yields

$$
\Re\left\langle\Psi \nu_{j} K_{j} \Psi\right\rangle=\left\langle\Psi \nu_{j}^{1 / 2},\left[K_{j}-\left|\frac{\nabla_{j} V_{j}}{2 V_{j}}\right|^{2}\right] \Psi \nu_{j}^{1 / 2}\right\rangle
$$

in accordance with the symmetry between $g$ and $g^{-1}$ noted above. Because, as discussed above, we will only be concerned with applications for which $\left\langle\Psi \nu_{j} K_{j} \Psi\right\rangle$ is real we will henceforth omit the $\Re$. We now discuss several cases in more detail using $\mathbf{d}$ to denote the dimension of the space on which $K_{j}$ acts. (The original proof of Lieb used an argument which originated with Benguria (see Lemma 7.20 of [12]) in the spherically symmetric case to show directly that $\Re\langle\nu \Psi(-\Delta) \Psi\rangle>0$. The variant given here is due to Hoffman-Ostenhof [9]. For other strategies see [5, 6, 10]) 
- $\mathbf{A}=\mathbf{0}, \mathbf{d} \geq \mathbf{3}$ : In this case we are interested in $\nu=[V(\mathbf{r})]^{-1}$ for potentials, (particularly the usual Coulomb potential $V(\mathbf{r})=1 /|\mathbf{r}|$ ) which satisfy $\left|\frac{\nabla V(\mathbf{r})}{V(\mathbf{r})}\right| \leq \frac{1}{|\mathbf{r}|}$. Then $K=-\Delta$ and (30) becomes

$$
\begin{aligned}
\left\langle\Psi \nu_{j} K_{j} \Psi\right\rangle & =\left\langle\Psi \nu_{j}\left(-\Delta_{j}\right) \Psi\right\rangle \\
& \geq\left\langle\Psi \nu_{j}^{1 / 2},\left[-\Delta_{j}-\frac{1}{4|\mathbf{r}|^{2}}\right] \Psi \nu_{j}^{1 / 2}\right\rangle \geq 0
\end{aligned}
$$

-A $\neq \mathbf{0}, \mathbf{d}=\mathbf{3}$ : In this case we can only conclude from (29)that

$$
\begin{aligned}
\left\langle\Psi \nu_{j} K_{j} \Psi\right\rangle & \geq B\left\|\Psi \nu_{j}^{1 / 2}\right\|^{2}-\left\langle\Psi \nu_{j}^{1 / 2},\left[\frac{\partial^{2}}{\partial x_{j}^{2}}-\left|\frac{\nabla_{j} \nu_{j}}{2 \nu_{j}}\right|^{2}\right] \Psi \nu_{j}^{1 / 2}\right\rangle \\
& \geq B\left\|\Psi \nu_{j}^{1 / 2}\right\|^{2}-\left\langle\Psi \frac{\left|\nabla_{j} \nu_{j}\right|^{2}}{4 \nu_{j}} \Psi\right\rangle .
\end{aligned}
$$

We could instead have proceeded as in (31) above to obtain, $\left\langle\Psi \nu_{j} K_{j} \Psi\right\rangle>0$, but we cannot conclude that $\left\langle\Psi \nu_{j} K_{j} \Psi\right\rangle \geq B\left\|\Psi \nu_{j}^{1 / 2}\right\|^{2}$. The kinetic energy $P_{x}^{2}=\frac{\partial^{2}}{\partial x_{j}^{2}}$ in the field direction is not able to control a 3-dimensional potential, such as $1 / \mathbf{r}^{2}$ arising from $\left|\frac{\nabla_{j} \nu_{j}}{2 \nu_{j}}\right|^{2}$. Our one-dimensional models circumvents this problem because the choice of product state involving Landau functions ensured that the $P_{y}^{2}+P_{z}^{2}$ terms took care of the $B$, leaving $P_{x}^{2}$ to deal with a one-dimensional potential $\left[V^{\prime}(x) / 2 V(x)\right]^{2}$ as described below.

- $\mathbf{A}=\mathbf{0}, \mathbf{d}=\mathbf{1}$ : In one dimension, $\mathbf{A}$ plays no role. Although we will still choose $\nu=[V(|r|)]^{-1}$ with potentials which satisfy $\left|\frac{V^{\prime}(x)}{x}\right|^{2}<\frac{1}{|x|^{2}}$ it is not true in one or two-dimensions that $-\Delta-|2 x|^{-2}>0$. Instead we will treat $-\left|\frac{V^{\prime}}{2 V}\right|^{2}$ as a potential in

$$
\left\langle\Psi \nu_{j}\left(-\Delta_{j}\right) \Psi\right\rangle=\left\langle\Psi \nu_{j}^{1 / 2},\left[-\frac{d^{2}}{d x_{j}^{2}}-\left|\frac{V^{\prime}\left(x_{j}\right)}{2 V\left(x_{j}\right)}\right|^{2}\right] \Psi \nu_{j}^{1 / 2}\right\rangle .
$$

Now in one-dimension the everywhere negative potential $-\left|\frac{V^{\prime}}{2 V}\right|^{2}$ will always give rise to a bound state of $-\frac{d^{2}}{d x^{2}}-\left|\frac{V^{\prime}}{2 V}\right|^{2}$. Thus we can only conclude that $\left\langle\Psi \nu_{j}\left(-\Delta_{j}\right) \Psi\right\rangle \geq-\epsilon\left\|\Psi \nu_{j}^{1 / 2}\right\|^{2}$ where $-\epsilon$ is the lowest eigenvalue of this operator. However, the bound

$$
\left\langle\Psi \nu_{j}\left(-\Delta_{j}\right) \Psi\right\rangle \geq-\omega\|\Psi\|^{2}
$$


where $\omega=\sup _{x}\left|\nu^{\prime}(x)\right|^{2} / 4 \nu(x)$ will be more useful. Notice that, unlike the 3-dimensional case where the lower bound is zero, when applying this result to $H_{N}=h(N, Z, M)$ we will need to take into account the fact the entire kinetic energy term is multiplied by $1 / M($ or $\sqrt{B})$.

\section{Bound on the Maximum Negative Ioniza- tion}

We now apply Lieb's strategy, which yields $N_{\max }(Z, 0)<2 Z+1$ for atoms without magnetic fields, to our one-dimensional models for systems in strong magnetic fields. We obtain the following result.

Theorem 1 The maximum number of electrons $N_{\max }$ for which a Hamiltonian $h\left(N, Z, B^{-1 / 2}\right)$ of the form (10) has a bound state satisfies $N_{\max }<$ $2 Z+1+c \sqrt{B}$ for some constant $c$.

In the interesting case $B=O\left(Z^{3}\right)$ (which is the boundary of the LSY hyperstrong limit region in [17]), this yields a bound of the form $N_{\max }<2 Z+c Z^{3 / 2}$, rather than a linear one of the form $N_{\max }<c Z$ or the expected optimal $N_{\max }<2 Z+o(Z)$.

We apply the strategy of Section 3 with $H_{N}=h(N, Z, M)$ and $\nu=1 / V$.

In this case, the analysis of expectations of $V_{\text {int }}^{j}$ is straightforward because $\left\langle\nu_{j}^{1 / 2} \Psi, V \nu_{j}^{1 / 2} \Psi\right\rangle=\langle\Psi, \Psi\rangle$.

$$
\begin{aligned}
\sum_{j}\left\langle\nu_{j}^{1 / 2} \Psi V_{\text {int }}^{j} \nu_{j}^{1 / 2} \Psi\right\rangle \\
=-Z \sum_{j}\|\Psi\|^{2}+\sum_{j} \sum_{k \neq j}\left\langle\Psi, 2^{-1 / 2} V\left(2^{-1 / 2}\left|x_{j}-x_{k}\right|\right) V\left(x_{j}\right)^{-1} \Psi\right\rangle \\
=-N Z\|\Psi\|^{2}+\sum_{j<k}\left\langle\Psi, 2^{-1 / 2} V\left(2^{-1 / 2}\left|x_{j}-x_{k}\right|\right)\left[V\left(x_{j}\right)^{-1}+V\left(x_{k}\right)^{-1}\right] \Psi\right\rangle .
\end{aligned}
$$

Thus, if the potential satisfies

$$
2^{-1 / 2} V\left(2^{-1 / 2}\left|x_{j}-x_{k}\right|\right)\left[V\left(x_{j}\right)^{-1}+V\left(x_{k}\right)^{-1}\right]>1,
$$

then

$$
\sum_{j}\left\langle\nu_{j}^{1 / 2} \Psi V_{\mathrm{int}}^{j} \nu_{j}^{1 / 2} \Psi\right\rangle \geq[-N Z+N(N-1) / 2]\|\Psi\|^{2}
$$


For the cut-off potential $V(x)=1 /(|x|+1)$, (36) follows easily from the triangle inequality since

$$
\begin{aligned}
2^{1 / 2}\left[V\left(2^{-1 / 2}|w-x|\right)\right]^{-1} & =|w-x|+2^{1 / 2} \\
& \leq|w|+2^{1 / 2}+|x|+2^{1 / 2}=[V(w)]^{-1}+\left[V(x)^{-1}\right]
\end{aligned}
$$

For the regularized potential $V_{0}(x)$ we will use instead the convexity of $\nu_{0}(x)=\left[V_{0}(x)\right]^{-1}$. (Because the proof [28, 27] of this essential fact, although elementary, is rather delicate and not readily accessible, we provide a sketch in the Appendix.) Thus we find,

$$
\begin{aligned}
{\left[V_{0}(w)\right]^{-1}+\left[V_{0}(x)^{-1}\right] } & \geq 2\left[V_{0}(|w-x| / 2)\right]^{-1} \\
& \geq 2^{1 / 2}\left[V_{0}\left(2^{-1 / 2}|w-x|\right]^{-1}\right.
\end{aligned}
$$

where the first inequality used $V_{0}(x)=V_{0}(|x|)$ as well as the convexity of $\nu_{0}(x)=\left[V_{0}(x)\right]^{-1}$ and the second inequality follows from

$$
\begin{aligned}
V_{m}\left(2^{-1 / 2} y\right) & =[m !]^{-1} \int_{0}^{\infty} \frac{t^{m} e^{-t}}{\sqrt{y^{2} / 2+t}} d t \\
& =2^{1 / 2}[m !]^{-1} \int_{0}^{\infty} \frac{t^{m} e^{-t}}{\sqrt{y^{2}+2 t}} d t \\
& \leq 2^{1 / 2} V_{m}(y)
\end{aligned}
$$

with $y=2^{-1 / 2}|w-x|$ and $m=0$.

Thus we can conclude that, for both the cut-off potential $V_{\text {cut }}(x)=$ $1 /(|x|+1)$ and the regularized potential $V_{0}(x)$, the one dimensional Hamiltonian $h(N, Z, M)$ satisfies

$$
\begin{aligned}
& \operatorname{Tr} \Gamma_{0} h(N, Z, M) \\
& \quad \geq E_{0}(N, Z, M) \operatorname{Tr} \Gamma_{0}+[-N Z+N(N-1) / 2-N \omega / M]\|\Psi\|^{2} .
\end{aligned}
$$

The second term in (39) will be positive if $(N-1) / 2>Z+\omega / M$. For both potentials, $\omega=\sup _{x}\left|\nu^{\prime}(x)\right|^{2} / 4 \nu(x)$ is given by $\lim _{x \rightarrow 0}\left|\nu^{\prime}(x)\right|^{2} / 4 \nu(x)$. This yields, $\omega_{\text {cut }}=0.25$ for the cut-off potential and $\omega_{0}=\pi^{-3 / 2}<0.18$ for the regularized potential $V_{0}(x)$. The latter follows from $\omega=\nu(0)^{3}=\pi^{-3 / 2}$ and the fact that, as shown in the Appendix, $\left|\nu^{\prime}(x)\right|^{2} / 4 \nu(x)=\nu(x)[\nu(x)-|x|]^{2}$ is decreasing for $x>0$. Thus, in both cases, the second term in (39) will be positive if $N>2 Z+1+1 / 2 M$. Since binding implies $\operatorname{Tr} \Gamma_{0} h(N, Z, M) \leq$ 
$E_{0}(N, Z, M) \operatorname{Tr} \Gamma_{0}$ and $M$ is proportional to $B^{-1 / 2}$, we have shown that one can find a constant $c$ such that our model one-dimensional system does not have bound states if $N \geq 2 Z+1+c \sqrt{B}$ or $N_{\max }<2 Z+1+c \sqrt{B}$.

Although this bound is not optimal, it should be remembered that we are analyzing a Hamiltonian with the structure

$$
h\left(N, Z, B^{-1 / 2}\right)=h\left(N-1, Z, B^{-1 / 2}\right)+B^{1 / 2} K_{j}+V_{\text {int }}\left(x_{j}\right)
$$

and that the factor $\sqrt{B}$ multiplying the kinetic energy is something of a two-edged sword. On the one hand, it raises the energy of the effective one-electron Hamiltonian $B^{1 / 2} K_{1}+\int V_{\text {int }}\left|\psi\left(x_{1}, x_{2}, \ldots, x_{N}\right)\right|^{2} d x_{2} \ldots d x_{N}$; on the other, it multiplies any error arising from the kinetic energy - whether using Lieb's strategy or the Ruskai-Sigal localization approach [6, 21, 22, 26] - by $\sqrt{B}$. Since such correction terms are typically negative, the result of such treatments is to perversely magnify the negative error associated with a positive term otherwise expected to raise the energy.

Acknowledgment It is a pleasure to thank Professor Pierre Duclos for many helpful discussions, including a critical observation about the form of the interaction, and for his hospitality during visits to the the Université de Toulon et du Var and the Centre Physique Théorique at Luminy-Marseille. We are also grateful to Professor Phillip Solovej for several helpful comments and to Professor Elisabeth Werner for communicating the results in [27] and the strategy used to prove inequality (43) in the Appendix. The second author $[\mathrm{MBR}]$ appreciated the hospitality at the Université de Reims and at Georgia Tech where parts of this work were done.

\section{A Convexity and Properties of $1 / V_{0}(x)$}

Because $\nu(x)=1 / V_{0}(x)$ is nearly linear (see Figure 1 ), the proof of its convexity is somewhat delicate. It will follow from the upper bound in the following pair of inequalities, which are of some interest in their own right.

$$
\frac{3 x+\sqrt{x^{2}+4}}{4}<\nu(x)<\frac{2 x+\sqrt{x^{2}+3}}{3} .
$$

We now restrict attention to $x>0$ and define

$$
g_{k}(x)=\frac{k}{(k-1) x+\sqrt{x^{2}+k}}
$$




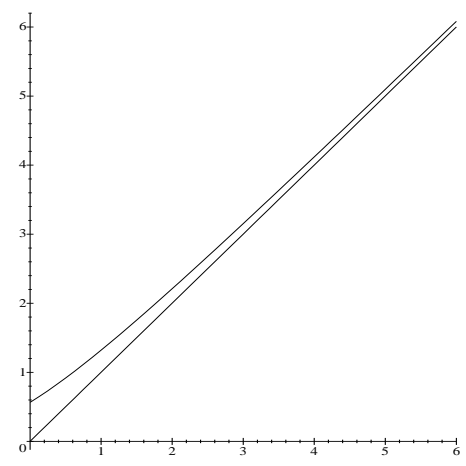

Figure 4: Comparison of $1 / V_{0}(x)$ and $x$ for $x \geq 0$.

so that (41) is equivalent to

$$
g_{4}(x)>V_{0}(x)>g_{3}(x)
$$

which is an improvement on the classical inequalities of Komatsu [11]. The upper bound and convexity were established independently by Wirth 28 and by Szarek and Werner [27]; the proof of lower bound which follows was communicated to the authors by E. Werner.

We now observe that $V_{0}(0)=\sqrt{\pi}$ and $V_{0}$ satisfies the differential equation

$$
V_{0}^{\prime}(x)=2\left[x V_{0}(x)-1\right]
$$

and look for analogous behavior for $g_{k}$. Since $g_{k}^{\prime}(x)=-\left[g_{k}(x)\right]^{2} \frac{x+(k-1) \sqrt{x^{2}+k}}{k \sqrt{x^{2}+k}}$ and $x g_{k}(x)-1=\frac{-1}{x+\sqrt{x^{2}+k}} g_{k}(x)$, one can verify that

$$
\begin{aligned}
g_{k}^{\prime}(x)> & 2\left[x g_{k}(x)-1\right] \\
& \Longleftrightarrow \frac{k}{\sqrt{x^{2}+k}} \frac{(k-1) \sqrt{x^{2}+k}+x}{(k-1) x+\sqrt{x^{2}+k}}<\frac{2 k}{x+\sqrt{x^{2}+k}} \\
& \Longleftrightarrow(k-2) x^{2}+k(k-3)<(k-2) x \sqrt{x^{2}+k} \\
& \Longleftrightarrow x^{2}(k-2)(k-4)+k(k-3)^{2}<0 .
\end{aligned}
$$

For $k=3$, we use the first equivalence to conclude from $x^{2}<x \sqrt{x^{2}+3}$ that $g_{3}^{\prime}(x)>2\left[x g_{3}(x)-1\right]$; for $k=4$, we use the second to conclude $k(k-3)^{2}>$ $0 \Rightarrow g_{4}^{\prime}(x)<2\left[x g_{4}(x)-1\right]$. 


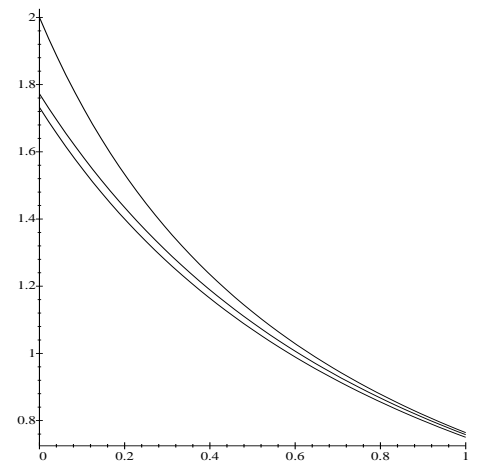

Figure 5: Graphs showing $g_{4}(x)>V_{0}(x)>g_{3}(x)$ for $x>0$.

For $k=3$, let $h_{3}(x)=V_{0}(x)-g_{3}(x)$. It is easily to verify that $h_{3}(0)=$ $\sqrt{\pi}-\sqrt{3}>0$ and that $\lim _{x \rightarrow \infty}\left|h_{3}(x)\right|=0$. The first equivalence in (45) implies that $x^{2}<x \sqrt{x^{2}+3} \Rightarrow g_{3}^{\prime}(x)>2\left[x g_{3}(x)-1\right]$ so that $h_{3}^{\prime}(x)<$ $2 x\left[V_{0}(x)-g_{3}(x)\right]=2 x h_{3}(x) \forall x$. Now suppose that for some $x=a, h_{3}(a)<0$. Then $h_{3}^{\prime}(a)<0$ and it follows that $h_{3}(x)$ is decreasing for $x>a$, which contradicts $\lim _{x \rightarrow \infty} h_{3}(x)=0$. This proves the lower bound in (43). The upper bound is proved similary [27] except that one now shows that $h_{4}(x)=$ $g_{4}(x)-V_{0}(x)>0$. It is interesting to note that the upper bound in (43) is optimal, but the lower bound can be improved [23] to $g_{\pi}(x)<V_{0}(x)$. The tightness of these bounds is evident in Figure 5 .

In order to use this to establish the convexity of $\nu(x)$, we note (44) implies that $\nu(x)=\left[V_{0}(x)\right]^{-1}$ satisfies

$$
\nu^{\prime}(x)=2 \nu(x)[\nu(x)-x] .
$$

Then

$$
\begin{aligned}
\nu^{\prime \prime}(x) & =4 \nu \nu^{\prime}-2 \nu^{\prime} x-2 \nu=4 \nu(\nu-x)(2 \nu-x)-2 \nu \\
& =8 \nu\left[\left(\nu-\frac{3}{4} x\right)^{2}-\frac{1}{16}\left(x^{2}+4\right)\right] \\
& =8 \nu\left[\left(\nu(x)-1 / g_{4}(x)+\frac{1}{4} \sqrt{x^{2}+4}\right)^{2}-\left(\frac{1}{4} \sqrt{x^{2}+4}\right)^{2}\right] .
\end{aligned}
$$

Since (41) implies $\nu(x)>1 / g_{4}(x)$, we can conclude that $\nu^{\prime \prime}(x)>0$.

To show that $\left|\nu^{\prime}(x)\right|^{2} / 4 \nu(x)=\nu(x)[\nu(x)-|x|]^{2}$ is decreasing for $x>0$, we note that (46) yields

$$
\frac{d}{d x}\left[\nu(\nu-x)^{2}\right]=2 \nu(\nu-x)\left[3 \nu^{2}-4 \nu x+x^{2}-1\right]
$$




$$
=6 \nu(\nu-x)\left[\left(\nu-\frac{2 x}{3}\right)^{2}-\frac{x^{2}+3}{9}\right]<0 .
$$

and (41) implies that the expression in square brackets is negative.

It is useful to have bounds on the extent to which $\nu(x)$ and $V_{0}(x)$ deviate from $|x|$ and the Coulomb potential, respectively, when $x$ is large. The upper bound in (41) implies $|\nu(x)-| x||<g_{4}(x)-x \leq 1 / 2|x|$ while the lower bound yields $\left|V_{0}(x)-1 /\right| x||<1 / x-g_{3}(x)<1 / 2 x^{3}$. The latter can also be proved directly from the integral representation (6).

\section{References}

[1] Y. Avron, I. Herbst, and B. Simon, "Schrödinger Operators with Magnetic Fields I. General Interactions" Duke Math. J. 45 847-883 (1978).

[2] Y. Avron, I. Herbst, and B. Simon, "Strongly Bound States of Hydrogen in Intense Magnetic Field" Phys. Rev. A 20, 2287-2296 (1979).

[3] Y. Avron, I. Herbst, and B. Simon "Schrödinger Operators with Magnetic Fields III. Atoms in Homogeneous Magnetic Field" Commun. Math. Phys. 79, 529-572 (1981).

[4] J.D. Baker, D.E. Freund, R.N. Hill and J.D. Morgan III "Radius of Convergence and Analytic Behavior of the 1/Z Expansion Phys. Rev. A 41, 1247-1273 (1990).

[5] B. Baumgartner, "On the Degree of Ionization in the TFW Theory" Lett. Math. Phys. 7, 439-441 (1983).

[6] H.L. Cycon, R.G. Froese, W. Kirsch, and B. Simon Schrödinger Operators (Springer-Verlag, 1987).

[7] C.L. Fefferman and L.A. Seco, "Asymptotic Neutrality of Large Ions" Commun. Math. Phys. 128, 109-130 (1990).

[8] I.K. Haines and D.H. Roberts, "One Dimensional Hydrogen Atom Am. J. Phys. 37, 1145-1154 (1969).

[9] T. and M. Hoffman-Ostenhof, private communication. 
[10] T. Ichinose, "Note on the Kinetic Energy Inequality Leading to Lieb's Negative Ionization Upper Bound" Lett. Math. Phys. 28, 219-230 (1993).

[11] K. Ito and H.P. McKean Diffusion Processes and Their Sample Paths (Springer-Verlag, 1965).

[12] E.H. Lieb, "Thomas-Fermi and Related Theories of Atoms and Molecules" Rev. Mod. Phys. 53, 603-641 (1981) in [14.

[13] E.H. Lieb, "Bound on the Maximum Negative Ionization of Atoms and Molecules" Phys. Rev. A 29, 3018-328 (1984) in [14].

[14] E.H. Lieb, The Stability of Matter: From Atoms to Stars Selecta of E. Lieb, ed. by W. Thirring (second edition, Springer-Verlag, 1997).

[15] E.H. Lieb, I.M. Sigal, B. Simon, and W. Thirring, "Asymptotic Neutrality of Large-Z Ions" Commun. Math. Phys. 116, 635-644 (1988) in 114.

[16] E.H. Lieb, J.P. Solovej and J. Yngvason, "Heavy Atoms in the Strong Magnetic Field of a Neutron Star" Phys. Rev. Lett. 69, 749-752 (1992).

[17] E.H. Lieb, J.P. Solovej and J. Yngvason, "Asymptotics of Heavy Atoms in High Magnetic Fields I: Lowest Landau Band Regions" Commun. Pure Appl. Math. 47, 513-591 (1993).

[18] E.H. Lieb, J.P. Solovej and J. Yngvason, "Asymptotics of Heavy Atoms in High Magnetic Fields II: Semiclassical Regions" Commun. Math. Phys. 161, 77-124 (1994).

[19] A.R.P. Rau, R.O. Mueller, and L. Spruch "Simple Model and Wave Functions for Atoms in Intense Magnetic Fields" Phys. Rev. A 11, 18651879 (1975).

[20] M. Ruderman, "Matter in Superstrong Magnetic Fields" Physics of Dense Matter C.J. Hansen, ed., pp. 117-131 (Rediel, Dordrecht, 1974).

[21] M.B. Ruskai, "Absence of Discrete Spectrum in Highly Negative Ions" Commun. Math. Phys. 82, 457-469; 85, 325-327 (1982). 
[22] M.B. Ruskai, "Improved Estimates on the Number of Bound States of Negative Bosonic Atoms" Ann. Inst. H. Poincare A: Physique Theorique 61, 153-162 (1994).

[23] M.B. Ruskai and E. Werner, "A Pair of Optimal Inequalities Related to the Error Function" preprint (Austin Math. Phys. Archive 97-564).

[24] L. Schiff and H. Snyder, "Theory of the Quadratic Zeeman Effect" Phys. Rev. 55, 59-63 (1939).

[25] L.A. Seco, I.M. Sigal, P. Solovej "Bound on the Ionization Energy of Large Atoms" Commun. Math. Phys. 131, 307-315 (1990).

[26] I.M. Sigal, "How Many Electrons Can a Nucleus Bind?" Ann. Phys. 157, 307-320 (1984).

[27] S. J. Szarek and E. Werner, "Confidence Regions for Means of Multivariate Normal Distributions and a Non-symmetric Correlation Inequality for Gaussian Measure" J. Multivariate Analysis (in press).

[28] M. Wirth, "On considère la fonction de $\mathbb{R}$ dans $\mathbb{R}$ défine par $f(x)=$ $e^{-x^{2} / 2}$; démontrer que la fonction $g$ de $\mathbb{R}$ dans $\mathbb{R}$ défine par $g(x)=$ $f(x) / \int_{x}^{\infty} f(t) d t$ est convexe" Revue de Mathématiques Spéciales 104, 187-88 (1993).

[29] J. Yngvason, "Asymptotics of Natural and Artificial Atoms in Strong Magnetic Fields" Proceeding of the XIth International Congress of Mathematical Physics D. Iagolnitzer, ed., pp. 185-205 (International Press, Cambridge, Massachusetts, 1995) in [14]. 